Pacific

Journal of

Mathematics

KNOT INVARIANTS IN 3-MANIFOLDS AND ESSENTIAL TORI

Paul Kirk and Charles Livingston

Volume $197 \quad$ No. 1

January 2001 


\title{
KNOT INVARIANTS IN 3-MANIFOLDS AND ESSENTIAL TORI
}

\author{
Paul Kirk And Charles Livingston
}

\begin{abstract}
Given a three-manifold $M$ and a cohomology class $\tau \in$ $H^{1}(M, Z / n Z)$, there is a naturally defined invariant of singular knots in $M$ with exactly one double point, $V_{\tau}$. It has been known that for some manifolds $V_{\tau}$ is integrable and that in these cases it defines an easily computed and highly effective knot invariant. This paper provides necessary and sufficient conditions on $M$ for the integrability of $V_{\tau}$. The class of manifolds for which $V_{\tau}$ is integrable (regardless of the choice of $\tau$ ) is shown to include all hyperbolic manifolds, all complements of knots in irreducible homology spheres, all irreducible $Z / 2 Z$-homology spheres, and most Seifert-fibered manifolds.
\end{abstract}

\section{Introduction.}

In $[\mathbf{K L}]$ we defined a new family of knot invariants (all of type 1) for knots in 3-manifolds. We then illustrated the usefulness and simplicity of these invariants for distinguishing knots and for addressing theoretical questions regarding unknotting operations. These invariants were shown to be welldefined for a large class of 3-manifolds, but we provided examples to show that our results did not extend to $S^{1} \times S^{2}$. Here we will give a complete description of the set of manifolds for which these knot invariants are welldefined.

Beyond gaining a clearer understanding of a particular class of invariants, our goal is to begin a detailed examination of the subtle interplay between the topology of a 3-manifold and its knot theory. For instance, past work, notably that of Kalfagianni [Ka], has demonstrated the utility of working in irreducible 3-manifolds; here the necessity of irreducibility is observed. More surprising is that more detailed 3-manifold structures play an equally central role. In particular, our main examples demonstrate that the invariants are not well-defined in most orientable 3 -manifolds that contain $S^{1}$ bundles over non-orientable surfaces. That such constraints already appear in working with type 1 invariants is surprising and begins to demonstrate the interplay between classical 3-manifold topology and the more recent approaches to knot theory. 
Another consequence of the results of this paper is that we provide a basis for extending the invariants of $[\mathbf{K L}]$ to knots in any oriented 3-manifolds; by taking the invariant in a certain quotient group it becomes well-defined. Indeed we will show that there is a homomorphism from the fundamental group of the free loop space on a 3-manifold $M$ to a free abelian group such that the invariants are well-defined in the cokernel of this homomorphism. Thus one can extend the topological applications of $[\mathbf{K L}]$ to arbitrary oriented 3-manifolds, with perhaps weaker conclusions.

Before we discuss the contents of this paper in detail, we will make a few comments about the subject of finite type invariants for knots in 3-manifolds.

The study of finite type invariants for knots in $S^{3}$ began with the work of Vassiliev on the stratification of the space of knots and has since grown in many directions. Generalizations to 3-manifolds other than $S^{3}$ have emerged from two different perspectives: Finite type invariants of 3-manifolds (such as the work of Ohtsuki [O]) and finite type invariants of knots in 3-manifolds (such as the work of Kalfagianni [Ka]). In both cases the type of an invariant roughly corresponds to its complexity.

In the first approach one views a 3-manifold as surgery on a framed link and the effect of Kirby moves is "factored out". The second setting considers invariants of knots in a 3-manifold whose extension to the space of singular knots vanishes on singular knots with more than $n$ crossings. In contrast to the first approach using surgery presentations, this point of view permits one to apply the important structure theorems in 3-manifold theory; notably the torus theorems of Jaco-Shalen and Johannson, and the solution of the Seifert conjecture of Casson-Jungreis and Gabai.

The problem solved in this paper is a complete determination of those manifolds for which a certain family of invariants of singular knots with one double point can be "integrated" to give a knot invariant. The singular knot invariants we study are simple and defined homologically and yet the structural properties of a 3-manifold affect whether the invariant can be integrated.

Notice that invariants constructed from this second point of view are somewhat unorthodox in knot theory. They are defined one free homotopy class at a time, and are secondary or relative invariants in the sense that they have no canonical normalizations. This corresponds to the fact that (except for the trivial homotopy class) there is no natural choice of "trivial" knot. Moreover, the group of type $n$ invariants need not be finitely generated; for example in $[\mathbf{K L}]$ we showed that the group of type 1 invariants for nullhomotopic knots in the solid torus is uncountable.

We briefly recall the construction of the invariants of $[\mathbf{K L}]$. Let $M$ be a compact, oriented 3-manifold. To each pair $(\tau, \gamma)$ where $\tau \in H^{1}(M ; \mathbf{Z} / n)$, and $\gamma$ is a free homotopy class in $M$ satisfying $\tau(\gamma)=0$, we constructed a function $V_{\tau, \gamma}\left(K_{t}\right) \in \mathbf{Z}[\mathbf{Z} / n]$ of homotopies $K_{t}$ with $K_{0}$ and $K_{1}$ embedded 
knots in the class $\gamma$. (For our purposes we view $\mathbf{Z}[\mathbf{Z} / n]$ as a free abelian group.)

This function vanishes on isotopies, and if it also vanishes on self-homotopies then we define

$$
v_{\tau, \gamma}\left(K_{0}, K_{1}\right)=V_{\tau, \gamma}\left(K_{t}\right) .
$$

Thus to make the knot invariants $v_{\tau, \gamma}\left(K_{0}, K_{1}\right)$ well-defined, we must show that $V_{\tau, \gamma}\left(K_{t}\right)$ vanishes if $K_{0}=K_{1}$. This requirement can be recast most succinctly in the following manner. Let $F M_{\gamma}$ denote the component of the free loop space on $M$ corresponding to the free homotopy class $\gamma$. By restricting to self-homotopies, $V_{\tau, \gamma}$ defines a homomorphism

$$
\bar{V}_{\tau, \gamma}: \pi_{1}\left(F M_{\gamma}\right) \longrightarrow \mathbf{Z}[\mathbf{Z} / n] .
$$

Then $v_{\tau, \gamma}\left(K_{0}, K_{1}\right)$ is well-defined if and only if $\bar{V}_{\tau, \gamma}$ is trivial. More generally $v_{\tau, \gamma}\left(K_{0}, K_{1}\right)$ is well-defined in the cokernel of $\bar{V}_{\tau, \gamma}$.

The purpose of this article is to investigate the homomorphism $\bar{V}_{\tau, \gamma}$ using techniques from classical 3-manifold topology. On the one hand, if this map is trivial, we obtain well-defined, easily computed, and useful knot invariants. If, however, the map is non-trivial, we obtain interesting classes in the first cohomology of the free loop space on $M$, and in general we get invariants of knots with values in the cokernel of $\bar{V}_{\tau, \gamma}$.

The main result is Theorem 4.2, which characterizes precisely those manifolds for which $\bar{V}_{\tau, \gamma}$ is zero for all $(\tau, \gamma)$. In Section 4 we define a class $\mathcal{M}$ of irreducible orientable 3 -manifolds which consists of those manifolds not containing the orientable circle bundle over the punctured Klein bottle, not containing certain embeddings of the orientable circle bundle over a punctured Möbius band, and also excluding the orientable Seifert fibered 3-manifolds which contain non-vertical tori. (See Section 4 for the precise definition of $\mathcal{M}$. We remark here that the class $\mathcal{M}$ includes all hyperbolic manifolds, all complements of knots in irreducible homology spheres, all irreducible $\mathbf{Z} / 2$-homology spheres, and most Seifert-fibered manifolds. However, it excludes some irreducible Haken manifolds.)

Theorem 4.2. Let $N$ be an oriented compact 3-manifold. The homomorphism

$$
\bar{V}_{\tau, \gamma}: \pi_{1}\left(F N_{\gamma}\right) \longrightarrow \mathbf{Z}[\mathbf{Z} / n]
$$

vanishes for all $\tau \in H^{1}(N ; \mathbf{Z} / n)$ and all free homotopy classes $\gamma$ satisfying $\tau(\gamma)=0$ if and only if $N$ is the connected sum of a manifold $P$ in $\mathcal{M}$ and a manifold $Q$ with trivial first integral homology.

In particular, the relative knot invariants $v_{\tau}\left(K_{0}, K_{1}\right)$ are well-defined for all $\tau$ and all homotopic pairs of knots $K_{0}, K_{1}$ in $N$ with $\tau\left(K_{i}\right)=0$ if and only if $N$ is the connected sum of a manifold in $\mathcal{M}$ and a manifold with trivial first homology. 
We now outline the contents of the paper. In Section 2 we recall the relevant definitions from $[\mathbf{K L}]$ and discuss the construction of the knot invariants $v_{\tau, \gamma}$ from $V_{\tau, \gamma}$. We explain how to interpret $V_{\tau, \gamma}$ as an equivariant intersection number in a cyclic cover of $M$.

We also explain how the homomorphisms $\bar{V}_{\tau, \gamma}$ yield interesting cohomology classes on the free loop space. Composing $\bar{V}_{\tau, \gamma}$ with any homomorphism (of abelian groups) $\mathbf{Z}[\mathbf{Z} / n] \longrightarrow \mathbf{Z}$ yields cohomology classes in $H^{1}\left(F M_{\gamma} ; \mathbf{Z}\right)$. We prove that these classes vanish on the based loop space $\Omega M_{\gamma}$ if $M$ is irreducible.

Section 3 forms the heart of this paper. We construct 5 types of "basic examples" of manifolds $M$ which admit pairs $(\tau, \gamma)$ with $\bar{V}_{\tau, \gamma}$ non-zero. In each case some aspect of the structure of $M$ is used to find $\tau$ and $\gamma$ for which $\bar{V}_{\tau, \gamma}$ is non-zero.

The first type of example arises when $M$ contains a non-separating 2sphere. The second type of example comes from a decomposition of $M$ as a connected sum of manifolds with non-trivial homology. The third (resp. fourth) type of example arises when $M$ contains a circle bundle over a punctured Klein bottle (resp. a circle bundle over a punctured Möbius band). Finally there are a few sporadic examples of closed irreducible Seifert fibered manifold which admit non-vertical incompressible tori; these too give examples with $\bar{V}_{\tau, \gamma}$ non-zero.

In Section 4 we prove the main result stated above. The theorems on incompressible tori in Haken 3-manifolds of Jaco-Shalen and Johannson as well as the solution of the Seifert conjecture by Casson-Jungreis and Gabai play a prominent role here. They are used to place a torus arising from a self-homotopy into a good position so that $\bar{V}_{\tau, \gamma}$ can be easily computed.

To close we would like to clarify the relationship of the present work with earlier work, especially that of $[\mathbf{K a}]$. Let $M$ be a closed, orientable, irreducible 3-manifold that is not a small Seifert fibered manifold and which does not contain a circle bundle over a non-orientable surface. In $[\mathbf{K a}]$ it is proved that any invariant of singular knots in $M$ that satisfies the "4-term relation" can be integrated to yield a knot invariant. (The results of $[\mathbf{K a}]$ extended those of Lin $[\mathbf{L i}]$, which applied in the case of simply connected manifolds.) Since the invariant we study here satisfies the 4-term relation, Kalfagianni's result can be applied to find a class of manifolds for which it is integrable. Here we describe the exact class of 3-manifolds for which our invariant is integrable; this class is larger than that given by the results of $[\mathbf{K a}]$. Of course, one of our main goals is to explore the necessity of the geometric constraints as well as their sufficiency.

We should also mention $[\mathbf{V}]$, in which a general theory of obstructions to the integrability of singular knot invariants is developed. It is observed there that certain geometric conditions on a 3-manifold can assure the vanishing of these integrability conditions. Irreduciblity is addressed in part by 
considering $S^{1} \times S^{2}$ (see also [KL]). The examples presented in this paper provide further examples of this interplay between the geometry of the 3 -manifold and the integrability of singular knot invariants.

\section{Preliminaries.}

We remind the reader of the construction of the invariants $v_{\tau}$ from $[\mathbf{K L}]$. These are Type 1 invariants in the sense that they vanish on all knots with 2 or more double points.

We consider triples $(M, \tau, \gamma)$, where:

(1) $M$ is a compact oriented 3-manifold,

(2) $\tau \in H^{1}(M ; \mathbf{Z} / n)$, viewed as a homomorphism from $H_{1}(M ; \mathbf{Z})$ to $\mathbf{Z} / n$, and

(3) $\gamma: S^{1} \longrightarrow M$ is a free homotopy class satisfying $\tau(\gamma)=0$.

Whenever $M$ is understood, an appropriate pair will refer to a pair $(\tau, \gamma)$ satisfying (2) and (3).

Definition 2.1. The term singular knot in $M$ means a smooth immersion of a circle into $M$ whose singularity set consists only of a finite number of double points such that the two vectors tangent to the circle at each double point are linearly independent.

Given a singular knot $K: S^{1} \longrightarrow M$ with one double point, we call the two oriented loops in the image of $K$ the lobes of $K$.

Definition 2.2. If $K_{0}$ and $K_{1}$ are homotopic embedded knots, a transverse homotopy from $K_{0}$ to $K_{1}$ is a homotopy $K_{t}, t \in[0,1]$, so that for $t \notin$ $\left\{t_{0}, t_{1}, \cdots, t_{n}\right\}, K_{t}$ is an embedding, and $K_{t_{i}}$ is a singular knot with one double point such that $K_{t_{i}+\epsilon}$ and $K_{t_{i}-\epsilon}$ are the two non-singular resolutions of $K_{t_{i}}$ (in some order) for small enough $\epsilon$. (A transverse homotopy always exists between any two homotopic knots.)

Given a triple $(M, \tau, \gamma)$ and a transverse homotopy $K_{t}: S^{1} \longrightarrow M$, $t \in[0,1]$ with $K_{0}$ and $K_{1}$ embedded, we define $V_{(M, \tau, \gamma)}\left(K_{t}\right) \in \mathbf{Z}[\mathbf{Z} / n]=$ $\mathbf{Z}\left[t, t^{-1}\right] /\left(t^{n}=1\right)$ to be the signed sum

$$
V_{(M, \tau, \gamma)}\left(K_{t}\right)=\sum_{i} \epsilon_{i}\left(t^{\tau\left(L_{1}(i)\right)}+t^{\tau\left(L_{2}(i)\right)}-2\right)
$$

where the sum is over the $i$ corresponding to the parameter $t_{i}$ when $K_{t_{i}}$ is singular, $L_{1}(i)$ and $L_{2}(i)$ denote the two lobes of the singular knot $K_{t_{i}}$, and $\epsilon_{i}$ denotes the sign of this crossing (i.e., the sign of the self-intersection number of the trace of the homotopy on $\left.\left(t_{i}-\delta, t_{i}+\delta\right)\right)$. For notational ease we will sometimes abbreviate $V_{(M, \tau, \gamma)}$ to $V_{\tau, \gamma}, V_{\tau}$, or $V$.

Let $A(n)$ denote the subgroup of $\mathbf{Z}[\mathbf{Z} / n]$ generated by elements of the form $t^{k}+t^{-k}-2$. By definition $V_{\tau}$ takes its values in $A(n)$. Notice that 
$A(n)$ is free abelian of rank $\left[\frac{n}{2}\right]$ if $n$ is non-zero and of infinite rank if $n$ is zero.

Lemma 2.3. The function $V_{\tau}$ of transverse homotopies extends to a welldefined function from the set of maps $K:[0,1] \longrightarrow \operatorname{Maps}\left(S^{1}, M\right)$ such that $K_{0}$ and $K_{1}$ are embedded knots in the class $\gamma$ to the abelian group $A(n)$. Moreover, $V_{\tau}$ is additive with respect to composition of paths and depends only on the homotopy class of $K$ rel endpoints.

In particular, the restriction of $V_{\tau}$ to self homotopies defines a homomorphism

$$
\pi_{1}\left(F M_{\gamma}\right) \longrightarrow A(n)
$$

where $F M_{\gamma}$ denotes the path component of the free loop space of $M$ corresponding to the class $\gamma$.

Proof. The only part of this which is not obvious is that $V_{\tau}\left(K_{t}\right)$ depends only on the homotopy class of $K_{t}$ rel the endpoints $K_{0}$ and $K_{1}$. This fact follows from the interpretation of $V_{\tau}\left(K_{t}\right)$ as an equivariant intersection number, as in Formula (2.3) below.

Notation. We will denote the restriction of $V_{(M, \tau, \gamma)}$ to self-homotopies as well as the homomorphism of Equation $(2.2)$ by $\bar{V}_{(M, \tau, \gamma)}: \pi_{1}\left(F M_{\gamma}\right) \longrightarrow$ $A(n)$. Depending on context this will be abbreviated to $\bar{V}_{\tau, \gamma}, \bar{V}_{\tau}$ or $\bar{V}$.

Remark. A convenient way to describe the construction given above is to define an invariant of singular knots with one crossing to be $f_{\tau}(K)=$ $t^{\tau\left(L_{1}\right)}+t^{\tau\left(L_{2}\right)}-2$ where the $L_{i}$ are the two lobes on $K$. The formula (2.1) defines the integral of $f_{\tau}$; this terminology comes from the fact that given an invariant $v$ of embedded knots one can derive an invariant $f$ for singular knots by the formula

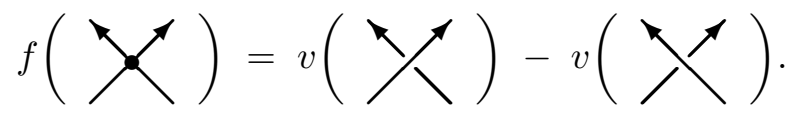

The invariants of $[\mathbf{K L}]$ are defined by inverting this process; thus given a pair of embedded knots $K_{0}$ and $K_{1}$ in the homotopy class $\gamma$ and a homotopy $K_{t}$ between them, define

$$
v_{(M, \tau, \gamma)}\left(K_{0}, K_{1}\right)=V_{(M, \tau, \gamma)}\left(K_{t}\right) .
$$

We will soon explore the extent to which this is well-defined. (Again we abbreviate this as $v_{\tau, \gamma}, v_{\tau}$, or $v$ as appropriate.)

In $[\mathbf{K L}]$ we showed that if $\tau$ is primitive, i.e., surjective as a homomorphism $H_{1}(M) \longrightarrow \mathbf{Z} / n$, then given any knot $K_{0}$ there exists a knot $K_{1}$ and a homotopy $K_{t}$ from $K_{0}$ to $K_{1}$ with $V_{\tau}\left(K_{t}\right)$ taking any prescribed value in the subgroup $A(n)$. 
Thus, as far as knot invariants are concerned, we conclude:

Theorem 2.4. Given a triple $(M, \tau, \gamma)$ and two knots $K_{0}, K_{1}$ in the class $\gamma$, a relative knot invariant $v_{\tau}\left(K_{0}, K_{1}\right) \in A(n) /$ Image $\bar{V}_{\tau, \gamma}$ is well-defined.

If $\tau$ is primitive then for any $K_{0}$ and any $a \in A(n) /$ Image $\bar{V}_{\tau, \gamma}$ there exists a knot $K_{1}$ homotopic to $K_{0}$ so that $v_{\tau}\left(K_{0}, K_{1}\right)=a$.

In particular, the image of the homomorphism $\bar{V}_{\tau, \gamma}: \pi_{1}\left(F M_{\gamma}\right) \longrightarrow A(n)$ provides an obstruction for $v_{\tau}\left(K_{0}, K_{1}\right)$ to be well-defined in $A(n)$. In Section 3 we will list all manifolds $M$ for which there exists an appropriate pair $(\tau, \gamma)$ with $\bar{V}_{\tau, \gamma}$ is non-trivial.

Remark. In $[\mathbf{K L}]$ we denoted $v_{\tau}\left(K_{0}, K_{1}\right)$ by $v_{\tau}\left(K_{0}\right)-v_{\tau}\left(K_{1}\right)$. We find the current notation more convenient since it eliminates the need to choose a base point. Some obvious formulas are

$$
v_{\tau}\left(K_{0}, K_{1}\right)+v_{\tau}\left(K_{1}, K_{2}\right)=v_{\tau}\left(K_{0}, K_{2}\right)
$$

and

$$
v_{\tau}\left(K_{0}, K_{1}\right)=-v_{\tau}\left(K_{1}, K_{0}\right) .
$$

Of course $v_{\tau}\left(K_{0}, K_{1}\right)=0$ if $K_{0}$ and $K_{1}$ are isotopic. These formulas are meant to be taken in $A(n) /$ Image $\bar{V}_{\tau, \gamma}$. One can obtain an invariant of knots (as opposed to pairs) by fixing a knot $K_{0}$ in the homotopy class $\gamma$ and then taking the invariant $K_{1} \mapsto v_{\tau}\left(K_{0}, K_{1}\right)$. This is the perspective taken in $[\mathrm{KL}]$.

We next turn to a discussion of the "intersection condition" from $[\mathbf{K L}]$. This is a reformulation of the map $\bar{V}_{\tau, \gamma}: \pi_{1}\left(F M_{\gamma}\right) \longrightarrow A(n)$ in terms of equivariant intersection numbers in the cyclic cover of $M$ determined by $\tau$. For what follows we assume that $\tau: H_{1}(M) \longrightarrow \mathbf{Z} / n$ with $n \neq 0$; the case $n=0$ can be understood by considering large values of $n$.

Choose the base point $K_{0} \in F M_{\gamma}$ to be any embedded knot in the class $\gamma$. A loop representing a class $\beta \in \pi_{1}\left(F M_{\gamma}, K_{0}\right)$ can be viewed as map $\beta^{\prime}$ : $T^{2} \longrightarrow M$ of a torus into $M$. Notice that $\tau$ vanishes on $\gamma$, and (since $n \neq 0$ ) by composing the loop $\beta$ with itself $n$ times if necessary, we may assume that $\beta^{\prime}$ pulls $\tau$ back to the zero class on the torus. Thus $\beta^{\prime}: T^{2} \longrightarrow M$ lifts to the $n$-fold cyclic cover $\tilde{M}$ of $M$ induced by $\tau$. Call this lift $\alpha: T^{2} \longrightarrow \tilde{M}$, and denote by $\alpha_{0}: S^{1} \longrightarrow \tilde{M}$ the restriction of this lift to the first factor; thus $\alpha_{0}$ is a lift of $K_{0}$.
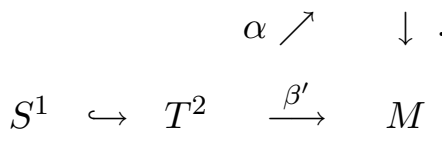
Notice that $V_{\tau}\left(\beta^{n}\right)=n \cdot V_{\tau}(\beta)$, and so for example $\bar{V}: \pi_{1}\left(F M_{\gamma}\right) \longrightarrow$ $\mathbf{Z}[\mathbf{Z} / n]$ is trivial if and only if $n \cdot \bar{V}$ is.

We confuse notation slightly and also let $\alpha \in H_{2}(\tilde{M}, \mathbf{Z})$ denote the homology class represented by $\alpha$ and $\alpha_{0} \in H_{1}(\tilde{M}, \mathbf{Z})$ denote the homology class represented by $\alpha_{0}$. With this notation we proved in $[\mathbf{K L}]$ that

$$
\bar{V}_{\tau, \gamma}(\beta)=\sum_{s=0}^{n-1}\left(\alpha \cdot\left(t^{s}+t^{-s}\right) \alpha_{0}\right)\left(t^{s}-1\right)
$$

in $\mathbf{Z}[\mathbf{Z} / n]$.

What this means is that we can construct elements in the image of $\bar{V}$ by constructing interesting examples of tori in $\tilde{M}$. Alternatively, $v_{\tau}$ gives a well-defined invariant if and only if this equivariant intersection number vanishes for all maps of tori $\alpha: T \longrightarrow \tilde{M}$ and all curves on such a torus.

As an application of this formula, we give more information about the homomorphism $\bar{V}_{\tau}: \pi_{1}\left(F M_{\gamma}\right) \longrightarrow A(n)$ when $M$ is an irreducible manifold. The free loop space $F M_{\gamma}$ maps to $M$ by evaluating a loop at $1 \in S^{1}$. The fiber of this map is the space $\Omega M_{\gamma}$ of based loops. The following theorem says that the homomorphisms $\bar{V}_{\tau, \gamma}$ vanish on $\pi_{1}\left(\Omega M_{\gamma}\right)$.

Theorem 2.5. Given a triple $(M, \tau, \gamma)$ as above, the composite

$$
\pi_{1}\left(\Omega M_{\gamma}\right) \longrightarrow \pi_{1}\left(F M_{\gamma}\right) \stackrel{\bar{V}}{\longrightarrow} A(n)
$$

vanishes whenever $\pi_{2}(M)=0$; for example if $M$ is irreducible.

Proof. As usual, it suffices to work with $n$ non-zero; the case of $n$ zero follows by considering large $n$.

Fix a knot $K$ in the homotopy class of $\gamma$ and let $\beta:(I, \partial I) \longrightarrow\left(\Omega M_{\gamma}, K\right)$ be a loop based at $K$. View $\beta$ as a map of a torus into $M, \beta^{\prime}: S^{1} \times S^{1} \longrightarrow M$. Notice that $\tau$ vanishes on both generators $S^{1} \times *$ and $* \times S^{1}$; indeed the first generator represents $\gamma$ and the restriction of $\beta^{\prime}$ to the second is the constant map. Thus $\beta^{\prime}$ lifts to $\alpha: T^{2} \longrightarrow \tilde{M}$, where $\tilde{M} \longrightarrow M$ denotes the $n$-fold cover determined by $\tau$. The lift $\alpha$ also restricts to the constant map on $* \times S^{1}$, and hence the homology class of $\alpha$ in $H_{2}(\tilde{M})$ is spherical. But $\pi_{2}(\tilde{M})=\pi_{2}(M)=0$, so that the homology class of $\alpha$ vanishes. From Formula 2.3 for $V_{\tau}$ given above one sees that $\bar{V}_{\tau}$ vanishes on $\beta$.

\section{The basic examples.}

In this section we will construct a number of examples of triples $(M, \tau, \gamma)$ with $\bar{V}_{(M, \tau, \gamma)}$ non-trivial. In the following section we will prove that the examples presented here form a complete list. The examples arise from 3 sources: Reducible manifolds, manifolds containing circle bundles over a non-orientable surface, and the few closed Seifert fibered 3-manifolds containing non-vertical tori. 


\section{A. Manifolds containing a punctured $S^{1} \times S^{2}$.}

Let $M$ be a once punctured $S^{1} \times S^{2}$. Let $\tau \in H^{1}(M ; \mathbf{Z})$ denote the Poincaré dual of the 2 -sphere, viewed as a homomorphism $\pi_{1} M \longrightarrow \mathbf{Z}$ and let $\tau_{n} \in H^{1}(M ; \mathbf{Z} / n)$ denote the reduction of $\tau$ modulo $n$ for some $n$ different from 0 or 1 . Let $\gamma_{n}$ denote the free homotopy class corresponding to a curve mapping to the circle with degree $n$; thus $\tau_{n}\left(\gamma_{n}\right)=0$.

The solid torus drawn in Figure 1 represents a neighborhood of the core circle and knots $K_{0}$ and $K_{1}$ in the class $\gamma_{n}$ are illustrated. (In the figure, $n=4$.) The homotopy $K_{t}$ given by the obvious crossing changes transform $K_{0}$ into $K_{1}$, showing that

$$
V_{\tau_{n}}\left(K_{t}\right)=\sum_{s=0}^{n-1}\left(t^{s}+t^{-s}-2\right) \neq 0 .
$$

Using the embedded sphere transverse to $\alpha$ one sees that $K_{0}$ and $K_{1}$ are isotopic. Thus the image of $\bar{V}: \pi_{1}\left(F M_{\gamma_{n}}\right) \longrightarrow A(n)$ is non-trivial for $\gamma_{n}$ the homotopy class corresponding to curves with winding number $n$.
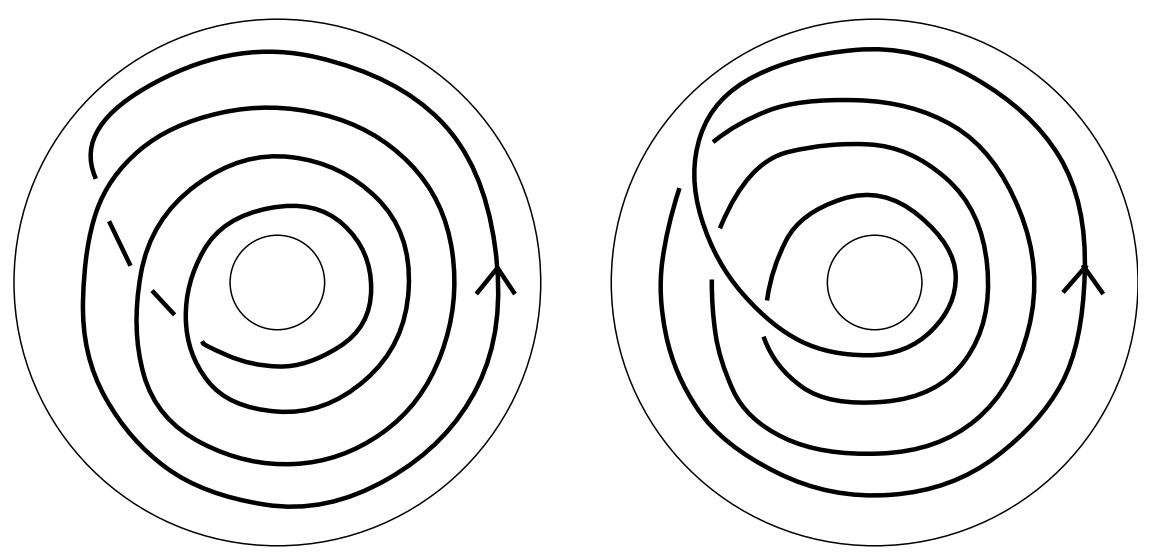

Figure 1.

Notice that the classes $\tau_{n}$ vanish on the boundary of $M$, and so extend to any manifold $N$ containing $M$. To summarize:

Proposition 3.1. Let $N$ be any 3-manifold containing a punctured $S^{1} \times$ $S^{2}$. Then for each $n \neq 0$ there exists a class $\tau_{n} \in H^{1}(N ; \mathbf{Z} / n)$ and a free homotopy class $\gamma_{n}$ with $\tau_{n}\left(\gamma_{n}\right)=0$ so that the image of the homomorphism

$$
\bar{V}_{\tau_{n}, \gamma_{n}}: \pi_{1}\left(F M_{\gamma_{n}}\right) \longrightarrow A(n)
$$

contains the subgroup spanned by the element $\sum_{s=0}^{n-1}\left(t^{s}+t^{-s}-2\right)$.

We conjecture that this element generates the image of $\bar{V}_{\tau_{n}, \gamma_{n}}$ for any $n$. 


\section{B. Connected sums of manifolds with non-trivial homology.}

We next show that if $M$ is the connected sum of two 3-manifolds each of which has non-trivial homology then there exists a pair $(\tau, \gamma)$ for which $\bar{V}$ is non-trivial.

Let $M$ be a connected sum $M=N_{1} \# N_{2}$ so that $H_{1}\left(N_{i} ; \mathbf{Z}\right) \neq 0$ for $i=1$ and 2. There exists an integer $n$ so that $H^{1}\left(N_{i} ; \mathbf{Z} / n\right)$ is non-zero for $i=1$ and 2 and thus one can find a class $\tau \in H^{1}(M ; \mathbf{Z} / n)$ whose restriction to $N_{1}$ and $N_{2}$ is non-trivial. Assume that $\tau$ is onto by replacing $\mathbf{Z} / n$ by the image of $\tau$ if necessary.

Let $\alpha$ be a loop in $N_{1}$ on which $\tau$ is non-trivial; say $\tau(\alpha)=a \in \mathbf{Z} / n-0$. Similarly choose a loop $\beta$ in $N_{2}$ with $\tau(\beta)=b \in \mathbf{Z} / n-0$. Pick $a \neq-b$; this is always possible unless $n=2$. If $n=2$ see below. Finally let $\gamma$ be the homotopy class in $M$ representing the commutator $\alpha \beta \alpha^{-1} \beta^{-1}$.

We can pick an embedded representative $K$ for $\gamma$ that meets the obvious separating 2 -sphere for the connected sum in exactly 4 points. One of the 4 strands that meets the 2 -sphere can be pushed around this 2 -sphere as in the previous example, yielding a self homotopy containing 3 singular knots. The value of $\bar{V}$ can be computed for this self homotopy, and the result is the non-trivial element $t^{a+b}-t^{b}+t^{a}-2+t^{-a}-t^{-b}+t^{-(a+b)}$ of $A(n)$.

If $n=2$, just take $\gamma$ to be the class represented by $\alpha \beta$. Note that $\tau$ takes the value $1 \in \mathbf{Z} / 2$ on $\alpha$ and $\beta$. Thus $\tau(\gamma)=\tau(\alpha)+\tau(\beta)=0$. An embedded representative for $K$ can be chosen which intersects the separating 2-sphere in two points. Pushing one strand around the 2 -sphere as before yields a self-homotopy with one double point. The value of $\bar{V}$ for this homotopy is $2 t-2 \neq 0$.

In this example the curve $\gamma$ is nullhomologous in $M$ (when $n \neq 2$ ). Contrast this with the fact proven in $[\mathbf{K L}]$ that for any $M$ and $\tau, \bar{V}_{\tau, \gamma}$ is zero whenever $\gamma$ is nullhomotopic, or even when $\gamma$ lies in the second commutator subgroup of $\pi_{1}(M)$.

We summarize these facts in the following proposition.

Proposition 3.2. Let $M$ be the connected sum of two 3-manifolds, $M_{1}$ and $M_{2}$, each of which has non-trivial homology. Then there exists an integer $n$ such that $H^{1}\left(M_{1} ; \mathbf{Z} / n\right)$ and $H^{1}\left(M_{2} ; \mathbf{Z} / n\right)$ are both non-zero and for any such $n$ there exists a pair $(\tau, \gamma)$ for which $\bar{V}_{\tau, \gamma}: \pi_{1}\left(F M_{\gamma}\right) \longrightarrow A(n)$ is non-trivial.

Moreover, if $n>2$, the curve $\gamma$ can be taken to be nullhomologous in $M$.

Remark. For many connected sums, for example for connected sums of manifolds in the class $\mathcal{M}$ defined below, one can compute the image of $\bar{V}_{\tau, \gamma}$ for most $\tau$ and $\gamma$.

Examples A. and B. reduce the problem of listing those 3-manifolds $M$ admitting a pair $(\tau, \gamma)$ with $\bar{V}$ non-zero to the case when $M$ is irreducible, or the connected sum of an irreducible manifold and a manifold with trivial 
first homology, i.e., a punctured homology sphere. We will show in the next section that taking the connected sum of $M$ with a punctured homology sphere does not affect whether or not the image of $\bar{V}$ is trivial.

Thus the remaining examples in this section are irreducible 3-manifolds which admit pairs $(\tau, \gamma)$ with $\bar{V}_{\tau, \gamma}$ non-trivial.

Examples $\mathrm{C}$ and $\mathrm{D}$ involve manifolds which contain certain circle bundles over non-orientable surfaces, and Example E involves a few exceptional closed Seifert fibered manifolds. The underlying motivation for studying these examples comes from the theory of torus decompositions of Haken manifolds ([JS $],[\mathbf{J} \mathbf{o}])$. In particular, the Seifert fibered components of the characteristic submanifold of a Haken manifold contain all the essential tori up to homotopy and so the search for examples with $\bar{V}$ non-zero is reduced to consideration of Seifert fibered manifolds.

We will see that except for the three 3-manifolds listed in Example E, the only way for an irreducible 3 -manifold to admit a pair $(\tau, \gamma)$ with $\bar{V}$ non-zero is if $M$ contains a Seifert fibration over a non-orientable surface. Conversely, most (but not all) 3-manifolds containing a Seifert fibration over a non-orientable base admit a pair $(\tau, \gamma)$ with $\bar{V}_{\tau, \gamma}$ non-zero.

\section{The twisted $S^{1}$ bundle over the punctured Klein bottle.}

Before focusing on particular $S^{1}$ bundles over surfaces, we need to make some general observations that will clarify the discussion in this and the next subsection. Let $F$ be an arbitrary connected non-orientable surface and let $M$ be the orientable $S^{1}$ bundle over $F$ formed as the union of two copies of the orientation $I$ bundle over $F$. There are two natural embeddings of $F$ into $M, h_{0}, h_{1}: F \rightarrow M$, each of which gives a section of the bundle. We denote the images by $F_{0}$ and $F_{1}$. Finally, there is a natural isomorphism $H_{1}(M, Z)=H_{1}\left(F_{0}\right) \oplus \mathbf{Z} / 2$; the second summand is generated by a circle fiber.

Let $h_{0}^{-1}$ denote the inverse of $h_{0}$ as a map defined on $F_{0}$. The embedding $h_{1} h_{0}^{-1}$ induces a map on homology, $h_{*}: H_{1}\left(F_{0}\right) \rightarrow H_{1}(M)$, satisfying $h_{*}(x)=(x, \epsilon(x)) \in H_{1}\left(F_{0}\right) \oplus \mathbf{Z} / 2$, where $\epsilon$ is the orientation character.

If $\gamma$ is a orientation preserving curve on $F_{0}$, then it is homotopic to $h_{1} h_{0}^{-1}(\gamma)$; the homotopy is obtained by sliding $\gamma$ along the fiber. However, if there is a double point on $\gamma$ splitting $\gamma$ into two lobes, each of which is orientation reversing, then the images of the lobes under $h_{1} h_{0}^{-1}$ are not homotopic to the original lobes. In fact, as the previous paragraph indicates, since the lobe $\lambda$ is orientation reversing, if it represents a class $x \in H_{1}\left(F_{0}\right)$ then it represents $(x, 0) \in H_{1}(M)$ while $h_{*}(\lambda)$ represents $(x, 1) \in H_{1}(M)$.

In Figure 2 below we illustrate the punctured Klein bottle $F$ and a curve $J$ on $F$. For the remainder of this paper let $M_{1}$ denote the twisted $S^{1}$ bundle over $F$ for which the total space is orientable. (There is a unique such $S^{1}$ bundle.) Note that $M_{1}$ is irreducible. 
For any $n$, and any $a \in \mathbf{Z} / n$, let $\tau: \pi_{1}\left(M_{1}\right) \longrightarrow \mathbf{Z} / n$ denote the character which factors through $\pi_{1}(F)$ and takes the values $a$ and $-a$ on the twisted bands illustrated in Figure 2.

If we consider $J$ to be an map of $S^{1}$ into $F$, then we can pull back the $S^{1}$ bundle over $F$ to $S^{1}$, and since $J$ is orientation preserving, this pullback bundle is trivial. Pick a section $K^{\prime}$ of this bundle. Its image in $M_{1}$, that we will denote by $K$, is a curve that projects to $J$ under the bundle projection. Furthermore we can assume that $K$ is embedded, since the only possible double point can be over the double point on $J$, and a small perturbation will remove any possible self intersection. Let $\gamma$ denote the free homotopy class of $K$.

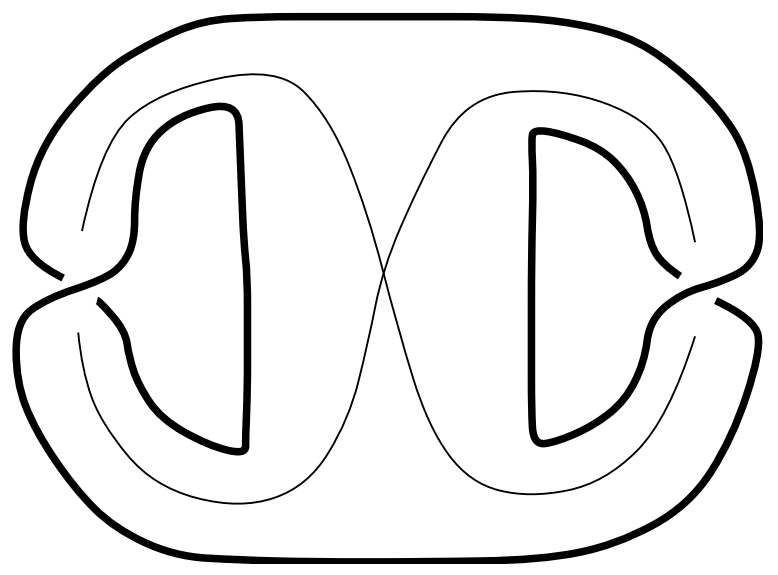

Figure 2.

There is a homotopy of $K^{\prime}$ to itself that pushes it once around the (trivial) fibration. This maps to a self homotopy of $K$ in $M_{1}$. Two points of $K$ lie above the double point of $J$, and during this homotopy the two points travel in opposite directions around the fiber. Hence, in the course of the homotopy there are two singular knots. The direction of crossing is the same on each, and the values of $\tau$ the lobes of these singular knots are $a$ and $-a$ in $\mathbf{Z} / n$. Hence, the value of $\bar{V}$ on this self homotopy is $2\left(t^{a}+t^{-a}-2\right)$.

Notice that the character $\tau$ used in this example restricts trivially to the boundary of $M_{1}$. Thus $\tau$ extends to every manifold containing $M_{1}$ and hence one obtains the following. (Note that $n=0$ is allowed in this proposition.)

Proposition 3.3. Every manifold $N$ containing $M_{1}$ admits a pair $(\tau, \gamma)$ so that the image of $\bar{V}_{\tau, \gamma}: \pi_{1}\left(F N_{\gamma}\right) \longrightarrow A(n)$ is non-trivial.

Notice that the image of $\bar{V}_{\tau, \gamma}: \pi_{1}\left(\left(F M_{1}\right)_{\gamma}\right) \longrightarrow A(n)$ is exactly the subgroup spanned by $2\left(t^{a}+t^{-a}-2\right)$. This can be seen as follows. Any map of a torus to $M_{1}$ is homotopic to a vertical torus. This is because $\pi_{1}(F)$ 
is free so any pair of commuting elements in $\pi_{1}\left(M_{1}\right)$ must map to a cyclic subgroup of $\pi_{1}(F)$ by the induced homomorphism $\pi_{1}\left(M_{1}\right) \longrightarrow \pi_{1}(F)$. Since $\gamma$ maps to the curve $J$ in $F$, The vertical torus must lie over $J$ up to homotopy. But then it is easy to see that a self-homotopy of $K$ is just a product of the self-homotopies of the same type as the one constructed above leading to a pair of double singular knots for each double point of the curve in $F$. In particular, the knot invariant $v_{\tau}\left(K_{0}, K_{1}\right)$ is well-defined in $A(n) /<2\left(t^{a}+t^{-a}-2\right)>\cong \mathbf{Z}^{[n / 2]-1} \oplus \mathbf{Z} / 2$.

Notice that the composite of this invariant with the projection to $\mathbf{Z} / 2$ gives a type 1 invariant which is not the reduction of a $v_{\tau}$. (Nevertheless, it turns out that for this example it is possible to construct a type 1 invariant with values in $\mathbf{Z}$ which reduces to this $\mathbf{Z} / 2$ invariant.)

There is a circle on the punctured Klein bottle intersecting $J$ exactly once. Since the bundle over the Klein bottle has a section the torus lying over $J$ has a dual curve, and so any 3 -manifold containing the twisted $S^{1}$ bundle over the punctured Klein bottle cannot be a rational homology sphere.

\section{The twisted $S^{1}$ bundle over the punctured projective plane.}

Any non-orientable surface which does not contain a punctured Klein bottle is homeomorphic to a $k$-punctured projective plane for some $k \geq 0$. Notice that there are exactly two circle bundles over the (unpunctured) projective plane with orientable total space. One of these is the lens space $L(4,1)$ which has a finite fundamental group, and hence $\bar{V}_{\tau, \gamma}$ vanishes for all appropriate pairs $(\tau, \gamma)$. The other one is homeomorphic to the connected sum of two $\mathbf{R P}^{3}$ s. This example gives the only non-prime orientable Seifert fibered space, and as Example B shows, it admits a pair $(\tau, \gamma)$ with $\bar{V}_{\tau, \gamma}$ non-zero.

We will show that if $M$ a circle bundle over the once-punctured projective plane, then $\bar{V}_{\tau, \gamma}$ is zero for all $(\tau, \gamma)$. However, if $M$ is a circle bundle over the $k$-punctured projective plane for $k>1$ then $\bar{V}_{\tau, \gamma}$ can be non-trivial for some $(\tau, \gamma)$.

Proposition 3.4. Let $N$ denote the orientable circle bundle over the Möbius band and let $M$ be an oriented compact manifold. Suppose an embedding $N \subset M$, a class $\tau \in H^{1}(M ; \mathbf{Z} / n)$, and a self-homotopy $K_{t}$ of a knot $K \subset M$ with $\tau([K])=0$ are given. If the map of the torus determined by $K_{t}$ is homotopic into $N$, then $\bar{V}_{\tau, \gamma}\left(K_{t}\right)=0$.

Proof. If the map of the torus into $N$ is compressible (i.e., not injective on $\pi_{1}$ ), then since $N$ is irreducible and has torsion free fundamental group, the homology class represented by this torus is spherical and thus trivial. Its lift, $\alpha$, to a finite cyclic cover of $N$ is also trivial and so the intersection numbers $\alpha \cdot t^{s} \alpha_{0}$ vanish. Hence $\bar{V}\left(K_{t}\right)=0$.

If the map is incompressible, then it is easy to see that the map of the torus is homotopic to a vertical map, and since any orientation preserving 
curve in the Möbius band is homotopic into the boundary, the torus itself is homotopic into the boundary of $N$. Thus any lift of the torus to a finite cyclic cover $\tilde{M}$ of $M$ is homotopic into the boundary of the inverse image $\tilde{N}$ of $N$. Interpreting $\bar{V}$ as an equivariant intersection number of a curve in the torus with the translates of the torus, one can easily use the collaring of $\partial \tilde{N}$ in $M$ to show that this intersection number vanishes.

We will show that if $M(k)$ denotes the orientable circle bundle over a $k$-punctured projective plane for $k>1$, then there do exist pairs $(\tau, \gamma)$ with $\bar{V}_{\tau, \gamma}$ non-trivial. However, not every manifold containing $M(k)$ admits such examples. For example the preceding proposition shows that $M(1)$ does not, even though it contains $M(k)$ for all $k>1$. We will give a specific criterion for which embeddings of $M(k)$ into a 3-manifold provide examples with $\bar{V}$ non-zero. It will turn out that if an embedding of $M(k)$ in a 3-manifold $M$ gives an example with $\bar{V}$ non-zero, then an embedding of $M(2)$ in the 3 -manifold already gives an example and so it suffices to test whether there are any "bad" embeddings of $M(2)$ in $M$.

We switch notation slightly; for the rest of this article we let $M_{2}$ denote the orientable $S^{1}$ bundle over the twice punctured projective plane. It is the union of the two orientable twisted $I$-bundles over the punctured Möbius band, and so contains the punctured Möbius band $F$ as a section. Fix such a section.

Figure 3 shows the punctured Möbius band $F$ and also a curve $J$ in $F$. This curve determines a vertical torus in $M_{2}$ as the union of fibers over $J$, and hence a self-homotopy of the knot in $M_{2}$ obtained by perturbing $J$ slightly along the fibers to be embedded.

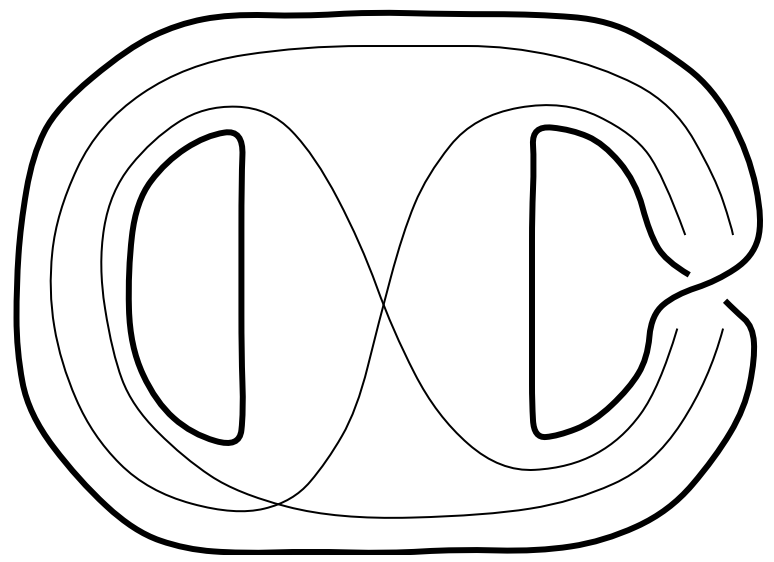

Figure 3. 
A simple computation shows that $H_{1}\left(M_{2} ; \mathbf{Z}\right)=\mathbf{Z} x \oplus \mathbf{Z} y \oplus \mathbf{Z} / 2 f$ where $x$ and $y$ are represented by curves going around the untwisted band and the twisted band in $F$ respectively, and $f$ is represented by the fiber in the fibration.

Let $\tau: \pi_{1}\left(M_{2}\right) \longrightarrow \mathbf{Z} / n$ be a homomorphism. Suppose that $\tau$ takes the value $a$ on the untwisted band and $b$ on the twisted band. Also suppose that $\tau$ takes the value $e$ on the fiber $f$; of course $e$ is zero if $n$ is odd or $n=0$, but for $n$ even $e$ can be either $n / 2$ or zero.

A similar analysis as in the case of $M_{1}$, using the comment in the first paragraphs of Subsection 3.C shows that the value of $\bar{V}_{\tau}$ on this self-homotopy is

$\left(t^{b}+t^{-b}-2\right)+\left(t^{b+e}+t^{-(b+e)}-2\right)-\left(t^{a+b}+t^{-(a+b)}-2\right)-\left(t^{a+b+e}+t^{-(a+b+e)}-2\right)$

in $\mathbf{Z}[\mathbf{Z} / n]$. This polynomial is non-zero except in the four cases: $a=0$, $2 b+a=0, a=e$, or $2 b+a=e$.

Remark. This example shows that the reduction mod 2 of $\bar{V}$ need not be zero. For example, take $n=8, a=b=1$ and $e=4$.

Notice that $\tau$ restricts non-trivially to both components of $\partial M_{2}$ if $a$ and $a+2 b$ are non-zero, or if $e$ is non-zero. In particular, given an embedding of $M_{2}$ in a manifold $M$, there may not exist any cohomology class on $M$ whose restriction to $M_{2}$ has all four $a, a+2 b, a+e$, and $2 b+a+e$ non-zero. With this in mind we make the following definition. Observe that the fibration of $M_{2}$ by circles induces a fibration of each of its two boundary tori.

Definition 3.5. An embedding $M_{2} \subset M$ is called simple if given any class $\tau \in H^{1}(M ; \mathbf{Z} / n)$, the restriction of $\tau$ to one of the boundary tori of $M_{2}$ vanishes on some curve intersecting the fiber transversally exactly once.

It is easy to check that an embedding is simple if and only if every $\tau \in$ $H^{1}(M ; \mathbf{Z} / n)$ restricts to $M_{2}$ so that one of $a, a+2 b, a-e$, and $2 b-a-e$ is zero. This immediately implies the following.

Proposition 3.6. If the oriented 3-manifold $M$ admits a non-simple embedding of $M_{2}$, then $M$ admits a pair $(\tau, \gamma)$ so that $\bar{V}_{\tau, \gamma}: \pi_{1}\left(F N_{\gamma}\right) \longrightarrow A(n)$ is non-zero.

The following theorem provides a converse to this statement which covers the cases of circle bundles over $k$-punctured projective planes for all $k>1$.

Theorem 3.7. Let $M$ be a 3-manifold and suppose that every embedding of $M_{2}$ into $M$ is simple. Given a pair $(\tau, \gamma)$, let $K_{t}$ be a self-homotopy of a knot in the homotopy class $\gamma$. Suppose that as a map of a torus, $K_{t}$ is homotopic into a submanifold of $M$ homeomorphic to the oriented circle bundle over a $k$-punctured projective plane for $k \geq 1$.

Then $\bar{V}_{\tau, \gamma}\left(K_{t}\right)=0$. 
Proof. The case when $k=1$ follows from Proposition 3.4. Suppose $M(k) \subset$ $M$ is an embedding containing $K_{t}$ for $k>1$. (Recall $M(k)$ denotes the oriented circle bundle over the $k$-punctured projective plane.) If a boundary component of $M(k)$ contains a curve dual to the fiber on which $\tau$ vanishes, then gluing in a solid torus to that boundary component in such a way that this curve bounds a disc yields a manifold over which both the fibering and $\tau$ extend; this manifold is just $M(k-1)$. Call a curve in a boundary component dual to the fiber on which $\tau$ vanishes $\tau$-reducible.

If all boundary components containing $\tau$-reducible curves are filled in, one obtains the manifold $M(q)$ for some $q \leq k$ and a class $\tau \in H^{1}(M(q) ; \mathbf{Z} / n)$ which does not vanish on any curve in $\partial M(q)$ dual to the fiber. If $q=1$, then Proposition 3.4 implies that $\bar{V}_{\tau}\left(K_{t}\right)=0$. If $q=0$ one need only fill in all but one boundary component of $M(k)$ to obtain $M(1)$; again Proposition 3.4 shows that $\bar{V}_{\tau}\left(K_{t}\right)=0$.

Thus we assume that there are at least two boundary components of $M(k)$ which do not contain $\tau$-reducible curves; this will lead to a contradiction in all but one special case where we will show directly that $\bar{V}_{\tau}\left(K_{t}\right)=0$.

The following argument is a bit technical so we begin by sketching the underlying idea. As in the previous examples, any generic orientation preserving curve on the $k$-punctured projective plane, $F(k)$, can be used to construct a knot with an interesting self-homotopy in the orientable circle bundle over $F(k), M(k)$. The value of a $\bar{V}_{\tau, \gamma}$ on that self-homotopy is determined by the value of $\tau$ on the lobes, and in general this leads to a complicated sum. However, note that $M(k)$ contains $M(2)$ in a variety of ways (since $F(k)$ contains $F(2)$ in many ways) and the fact that each of these is simple greatly constrains the possible values of $\tau$ on the various lobes. The details of the argument call on careful bookkeeping that we now undertake.

Fix a section $s: F(k) \longrightarrow M(k)$ of the fibration $M(k) \longrightarrow F(k)$ and identify $F(k)$ with its image. Label the boundary components of $F(k)$ as $C_{1}, C_{2}, \cdots, C_{k}$ and assume the numbering is chosen so that

$$
\tau\left(C_{i}\right)= \begin{cases}a_{i} \in \mathbf{Z} / n-\{0\} & \text { for } i=1,2, \ldots, \ell, \text { and } \\ 0 & \text { for } i=\ell+1, \ldots k .\end{cases}
$$

A collection of $k-2 \operatorname{arcs}$ in $F(k)$ which cuts $F(k)$ into $F(2)$ determines an embedding of $F(2) \subset F(k)$ and hence an embedding $M(2) \subset M(k) \subset M$. Since every embedding of $M_{2}$ in $M$ is simple, by cutting along appropriate arcs and using the fact that $F(k)$ is non-orientable, we can arrange that for any choice of signs $\epsilon_{i} \in\{ \pm 1\}, i=1, \cdots \ell$, and any partition $J \cup J^{c}=$ $\{1,2, \ldots, \ell\}$ one of the two sums

$$
\sum_{j \in J} \epsilon_{j} a_{j}, \quad \sum_{j \in J^{c}} \epsilon_{j} a_{j}
$$


is zero. An elementary argument implies that since $\ell \geq 2,2 a_{j}$ is zero for each $j=1, \ldots, \ell$. This is a contradiction if $n$ is odd or $n=0$.

Thus we assume $n$ is even and non-zero. Each $a_{j}$ equals $n / 2$. If $\ell$ is even, then the pair of sums in $(*)$ for $J=\{1\}$ reduces to $n / 2,(\ell-1) n / 2$, neither of which is zero; again a contradiction.

This leaves the case when $\ell$ is odd. We will show that in this case $\bar{V}_{\tau}\left(K_{t}\right)=0$. View $F(k)$ as a disk with one twisted and $k-1$ untwisted bands attached. Label the corresponding generators of $H_{1}(F(k) ; \mathbf{Z})$ represented by loops which travel once around the band $y$ and $x_{1}, \cdots x_{k-1}$. Then (with the $x_{i}$ and $y$ properly oriented) the boundary components of $F(k)$ are represented by the homology classes $x_{1}, \cdots, x_{k-1}$ and $2 y+\sum_{i} x_{i}$. Thus the (unordered) set

$$
\left\{\tau\left(x_{1}\right), \cdots, \tau\left(x_{k-1}\right), 2 \tau(y)+\sum_{i} \tau\left(x_{i}\right)\right\}
$$

contains $\ell$ repetitions of $n / 2$ and $k-\ell$ repetitions of 0 since this is just the set of $a_{j}$ and 0 . Using the fact that $\ell$ is odd it is easy to show that this implies that $n$ is divisible by 4 and $\tau(y)= \pm n / 4$.

Homotop the map of the torus given by $K_{t}$ to be vertical; in fact assume this torus lies over a self-transverse immersed orientation preserving curve $J$ in $F(k)$. This is possible since $M(k)$ contains no horizontal tori.

The double points of $J$ can be separated into two sets. The essential double points are those so that either (and hence both) of the loops obtained by starting at the double point and traveling along $J$ until one returns to this double point is orientation reversing. The other double points of $J$ are called inessential double points.

An argument just as in Example C shows that for this homotopy,

$$
\begin{aligned}
\bar{V}_{\tau}\left(K_{t}\right) & =\sum_{p \text { essential }} \epsilon_{p}\left(t^{n / 4}+t^{3 n / 4}+t^{e+n / 4}+t^{e+3 n / 4}-4\right) \\
& =2\left(t^{n / 4}+t^{3 n / 4}-2\right) \sum_{p \text { essential }} \epsilon_{p}
\end{aligned}
$$

where $e=\tau(f)=0$ or $n / 2$ and $\epsilon_{p} \in\{ \pm 1\}$ is the sign of the intersection corresponding to $p$.

Thus to finish the argument it suffices to show that the sum of the signs of the essential double points is zero. This is a statement about double points of orientation preserving immersed curves in a punctured projective plane and has nothing to do with $\tau$. In particular, it is true if and only if it is true in a (unpunctured) projective plane, since the orientable circle bundle over $F(k)$ extends to an orientable circle bundle over the projective plane. Any such curve in a projective plane is nullhomotopic and hence regularly 
homotopic to a curve with no essential double points. A generic regular homotopy pairs up the essential double points in pairs with canceling signs.

Thus the sum of the $\epsilon_{p}$ over the essential double points is zero, and so $\bar{V}_{\tau}\left(K_{t}\right)$ vanishes as claimed.

Notice that $M_{2}$ is irreducible and has first homology $H_{1}\left(M_{2} ; \mathbf{Z}\right)=\mathbf{Z}^{2} \oplus$ $\mathbf{Z} / 2$. From this it follows easily that $M_{2}$ is not contained in any $\mathbf{Z} / 2$ homology sphere, or even in any rational homology sphere with $X$ with $H_{1}(X ; \mathbf{Z})=\mathbf{Z} / 2 \oplus T$ for $T$ an odd torsion abelian group.

\section{E. The Seifert fibered spaces containing horizontal tori.}

We showed in $[\mathbf{K L}]$ (see also Section 4 ) that if $K_{t}$ is a self-homotopy of a knot in a Seifert fibered manifold $M$ with orientable base, then $\bar{V}\left(K_{t}\right)$ vanishes provided that $K_{t}$ (viewed as a map of a torus) is homotopic to a vertical map. In light of the characteristic submanifold theorem of $[\mathbf{J S}]$ and $[\mathbf{J o}]$, as well as the main theorem of $[\mathbf{G}]$ and $[\mathbf{C J}]$, the search for 3-manifolds $M$ which admit $(\tau, \gamma)$ with $\bar{V}_{\tau, \gamma}$ non-trivial is reduced to the examples containing non-vertical tori.

There are only a few irreducible orientable Seifert fibered 3-manifolds which admit non-vertical maps of tori. Two of these have no singular fibers, namely the three torus $T^{3}$ and the orientable circle bundle over the Klein bottle with Euler class zero. In $T^{3}$, any incompressible torus is homotopic to a vertical torus in some fibration over $T^{2}$. Any oriented circle bundle over the Klein bottle contains $M_{1}$ and so Example $\mathrm{C}$ above shows that it admits a pair $(\tau, \gamma)$ with $\bar{V}_{\tau, \gamma}$ non-trivial. There remain five examples; these have singular fibers.

Let $M\left(S^{2},(3,1),(3,1),(3,-2)\right)$ be the unique Seifert fibered space over $S^{2}$ with three singular fibers and Seifert invariants $(3,1),(3,1)$, and $(3,-2)$. Equivalently, this is the unique (up to orientation) Seifert fibration over $S^{2}$ with 3 singular fibers each of type 3 , and with Euler class equal to zero. For brevity we will denote this manifold $M_{(3,3,3)}$.

Similarly let $M_{(2,4,4)}$ denote $M\left(S^{2},(2,1),(4,-1),(4,-1)\right)$, let $M_{(2,3,6)}$ denote $M\left(S^{2},(2,1),(3,-1),(6,-1)\right)$, and let $M_{(2,2,2,2)}$ denote the Seifert fibered space over $S^{2}$ with four singular fibers $M\left(S^{2},(2,1),(2,1),(2,-1)\right.$, $(2,-1))$. Finally, let $M_{(2,2)}$ denote the orientable Seifert fibered space over the projective plane with two singular fibers and Euler class zero, i.e., $M_{(2,2)}=M\left(\mathbf{R} P^{2},(2,1),(2,-1)\right)$.

The manifolds $M_{(3,3,3)}, M_{(2,4,4)}, M_{(2,3,6)}, M_{(2,2,2,2)}$ are the four non-trivial orientable torus bundles over the circle with finite order monodromy, and $M_{(2,2,2,2)}$ is a 2 -fold cover of $M_{(2,2)}$. These five form a complete list of Seifert fibrations of orientable manifolds which have singular fibers and which contain non-vertical essential tori. 
It is not hard to identify $M_{(2,2,2,2)}$ with the orientable circle bundle over the Klein bottle (the identification is not fiber preserving) and so this manifold contains $M_{1}$. From Example $\mathrm{C}$ we know that there are appropriate pairs $(\tau, \gamma)$ with $\bar{V}_{\tau, \gamma}$ non-trivial.

Also notice that $M_{(2,2)}$ contains $M_{2}$ as the complement of the singular fibers. This embedding is non-simple; one can easily find a class $\tau \in$ $H^{1}\left(M_{(2,2)} ; \mathbf{Z} / 2\right)$ whose restriction to $M_{2}$ has $a$ and $a+2 b$ non-zero. From Example D we know that there exists a pair $(\tau, \gamma)$ in $M_{(2,2)}$ with $\bar{V}_{\tau, \gamma}$ nonzero.

The remaining examples, $M_{(2,3,6)}, M_{(2,4,4)}$, and $M_{(3,3,3)}$ do not contain a circle bundle over a non-orientable surface. We will show that nevertheless these admit a pair $(\tau, \gamma)$ so that $\bar{V}_{\tau, \gamma}$ is non-zero. The corresponding map of a torus necessarily will be an incompressible, non-vertical torus.

The three manifolds $M_{(2,3,6)}, M_{(2,4,4)}$, and $M_{(3,3,3)}$ have the structure of torus bundles over the circle with finite order monodromy. The monodromy maps for $M_{(2,3,6)}, M_{(2,4,4)}$, and $M_{(3,3,3)}$ are given respectively by the linear maps $\mathbf{R}^{2} / \mathbf{Z}^{2} \longrightarrow \mathbf{R}^{2} / \mathbf{Z}^{2}$ determined by the matrices

$$
A=\left(\begin{array}{cc}
0 & -1 \\
1 & 1
\end{array}\right), \quad B=\left(\begin{array}{rr}
0 & 1 \\
-1 & 0
\end{array}\right), \quad \text { and } C=\left(\begin{array}{ll}
0 & -1 \\
1 & -1
\end{array}\right) .
$$

We will find a pair $(\tau, \gamma)$ in $M_{(3,3,3)}$ with $\bar{V}_{\tau, \gamma}$ non-trivial; similar linear algebra gives examples in $M_{(2,3,6)}, M_{(2,4,4)}$, and $M_{(2,2,2,2)}$.

The monodromy matrix $C$ has order 3 . Thus the torus bundle over $S^{1}$ with monodromy $C^{3}$ is just $T^{3}$ and is a 3 -fold cyclic cover of $M_{(3,3,3)}$. It is easy to check that the covering transformation $t: T^{3} \longrightarrow T^{3}$ induces the isomorphism on $H_{1}(T)=\mathbf{Z}^{3}$ given by the matrix

$$
t=\left(\begin{array}{lll}
1 & 0 & 0 \\
0 & C \\
0 & C
\end{array}\right) .
$$

Let $\alpha: T^{2} \longrightarrow T^{3}$ be the map $\alpha\left(e^{i x}, e^{i y}\right)=\left(e^{i x}, e^{i x}, e^{i y}\right)$. Let $\alpha_{0}$ be the restriction of $\alpha$ to $S^{1} \times\{1\}$, so $\alpha_{0}\left(e^{i x}\right)=\left(e^{i x}, e^{i x}, 1\right)$. Then

$$
\begin{aligned}
\left(t+t^{-1}\right) \alpha_{0} \cdot \alpha & =\operatorname{det}\left(\left(t+t^{-1}\right)\left(\begin{array}{l}
1 \\
1 \\
0
\end{array}\right) \begin{array}{ll}
1 & 0 \\
1 & 0 \\
0 & 1
\end{array}\right) \\
& =3 .
\end{aligned}
$$

Letting $\tau \in H^{1}\left(M_{(3,3,3)} ; \mathbf{Z} / 3\right)$ denote the character defining this three fold cover and denoting by $\gamma$ the homotopy class of the knot $S^{1} \stackrel{\alpha_{0}}{\longrightarrow} T^{3} \longrightarrow M_{(3,3,3)}$ the composite $T^{2} \stackrel{\alpha}{\longrightarrow} T^{3} \longrightarrow M_{(3,3,3)}$ defines a self-homotopy $K_{t}$ with

$$
\bar{V}_{\tau, \gamma}\left(K_{t}\right)=3 t+3 t^{-1}-6 .
$$




\section{The class of admissible manifolds.}

In this section we prove our main result characterizing those manifolds $M$ so that $v_{\tau}$ is well-defined for all appropriate pairs $(\tau, \gamma)$.

Definition 4.1. Let $\mathcal{M}$ denote the class of all orientable compact irreducible 3-manifolds excluding:

1. Any 3-manifold containing a circle bundle over the punctured Klein bottle,

2. any 3-manifold containing a non-simple embedding of the orientable circle bundle over the twice-punctured projective plane, and

3 . the three manifolds $M_{(3,3,3)}, M_{(2,3,6)}$, and $M_{(2,4,4)}$.

Theorem 4.2. Let $N$ be an oriented compact 3-manifold. The homomorphism

$$
\bar{V}_{\tau, \gamma}: \pi_{1}\left(F N_{\gamma}\right) \longrightarrow A(n)
$$

vanishes for all $\tau \in H^{1}(N ; \mathbf{Z} / n)$ and all free homotopy classes $\gamma$ satisfying $\tau(\gamma)=0$ if and only if $N$ is the connected sum of a manifold $P$ in $\mathcal{M}$ and a manifold $Q$ with trivial first integral homology.

In particular, the relative knot invariants $v_{\tau}\left(K_{0}, K_{1}\right)$ are well-defined for all $\tau$ and all homotopic pairs of knots $K_{0}, K_{1}$ in $N$ with $\tau\left(K_{i}\right)=0$ if and only if $N$ is the connected sum of a manifold in $\mathcal{M}$ and a manifold with trivial first homology.

We essentially proved sufficiency in $[\mathbf{K L}]$. The result in that article gives a slightly weakened form of the following lemma.

Lemma 4.3. Let $M \in \mathcal{M}$. Suppose that $\tilde{M} \longrightarrow M$ is an $n$-fold cyclic covering of $M$ with covering transformation generated by $t: \tilde{M} \longrightarrow M$. Let $\alpha: T^{2} \longrightarrow \tilde{M}$ be an essential map of a torus; i.e., $\alpha$ is injective on fundamental groups and $\alpha$ is not homotopic into the boundary of $\tilde{M}$. Let $\alpha_{0}: S^{1} \longrightarrow \tilde{M}$ denote the restriction of $\alpha$ to the first factor $S^{1} \subset T^{2}=$ $S^{1} \times S^{1}$. Then the intersection number

$$
\left(t^{s}+t^{-s}\right) \alpha \cdot \alpha_{0}
$$

vanishes.

Proof. The proof of this lemma is essentially the same as the proof of Lemma 4.5 of $[\mathbf{K L}]$; the only case not covered in that article is the following.

Let $\beta: T^{2} \longrightarrow M$ denote the composite of $\alpha$ and the covering projection. Assume that $\beta$ is an essential map (injective on $\pi_{1}$ and not homotopic into the boundary of $M$ ). Assume moreover that $\beta$ is homotopic into a vertical torus in a Seifert fibered submanifold $C \subset M$ and that $C$ is Seifert-fibered over a non-orientable surface.

Then Proposition 3.4 and Theorem 3.7 imply that

$$
0=\bar{V}(\beta)=\left(t^{s}+t^{-s}\right) \alpha \cdot \alpha_{0} .
$$


All other cases follow exactly as in the proof of Lemma 4.5 of $[\mathbf{K L}]$.

Proof of Theorem 4.2. Assume that $N$ has non-trivial first homology, otherwise the statements are all trivially true.

If $\bar{V}_{\tau, \gamma}$ vanishes for all $(\tau, \gamma)$, consider a decomposition of $N$ as a connected sum $N=P \# Q$ with $P$ prime such that $H_{1}(P ; \mathbf{Z}) \neq 0$. Then Example A of Section 3 shows that $P$ is not $S^{1} \times S^{2}$; hence $P$ is irreducible. Example B shows that $Q$ must have trivial first homology, $H_{1}(Q ; \mathbf{Z})=0$.

If $P$ is not in $\mathcal{M}$, then either $P$ contains $M_{1}$, a non-simple $M_{2}$, or $P$ is one of $M_{(3,3,3)}, M_{(2,3,6)}$, or $M_{(2,4,4)}$. The remaining examples of Section 3 show that in any of these cases one can find an appropriate pair $(\tau, \gamma)$ such that $\bar{V}_{\tau, \gamma}$ is non-trivial. Notice that $\tau$ extends over $N=P \# Q$ and that $\gamma$ can be viewed as a free homotopy class in $N$. Moreover, any map of a torus into $P$ can be pushed off a neighborhood of a point and so $\bar{V}_{\tau, \gamma}$ is non-trivial in $N$. This contradiction means that $P \in \mathcal{M}$, as claimed.

For the converse, suppose that $N=P \# Q$ where $P \in \mathcal{M}$ and $H_{1}(Q ; \mathbf{Z})=$ 0 . Let $\tau \in H_{1}(P ; \mathbf{Z} / n)$ and $K_{t}$ a self-homotopy of a knot $K_{0}$ with $\tau\left(K_{0}\right)=0$. To show that $\bar{V}_{\tau}\left(K_{t}\right)=0$ it suffices to prove this for $n \neq 0$ by taking the reduction of an integer class modulo $n$ for large enough $n$ if necessary. Also, as explained above, we may assume that the self-homotopy $K_{t}$ lifts to the $n$-fold cyclic cover $\tilde{N} \longrightarrow N$ determined by $\tau: H_{1}(N) \longrightarrow \mathbf{Z} / n$.

Sticking to our notation we denote by $\beta: T^{2} \longrightarrow P$ the map given by viewing the self-homotopy of $K_{0}$ as a map of the torus, and we denote the lift of $\beta$ to $\tilde{N}$ by $\alpha: T^{2} \longrightarrow \tilde{N}$. Its restriction to the lift of the knot is denoted by $\alpha_{0}: S^{1}=S^{1} \times * \longrightarrow \tilde{N}$. What must be shown is that for each $s \neq 0$, the intersection number $\left(t^{s}+t^{-s}\right) \alpha_{0} \cdot \alpha$ vanishes, where $t: \tilde{N} \longrightarrow \tilde{N}$ denotes the generator of the covering transformations.

Let $P_{0}$ and $Q_{0}$ denote the once punctured $P$ and $Q$, and let $S \subset N$ denote the 2-sphere separating $P_{0}$ from $Q_{0}$ so that $N$ is the union of $P_{0}$ and $Q_{0}$ along $S$. The preimage of $S$ in $\tilde{N}$ consists of $n 2$-spheres which we denote by $\tilde{S}, t \tilde{S}, t^{2} \tilde{S}, \cdots, t^{s-1} \tilde{S}$. These 2 -spheres separate $\tilde{N}$ into $\tilde{P}_{0}$ and $n$ disjoint copies of $Q_{0}$ translated cyclicly by the covering translations. This is because $\tau$ vanishes on $H_{1}\left(Q_{0}\right)$. Figure 4 illustrates the decomposition $\tilde{N}=\tilde{P}_{0} \cup_{s} t^{s} Q_{0}$.

By a homotopy of $\beta$ we may assume that $\beta$ is transverse to $S$ and hence $\alpha$ is transverse to $t^{s} \tilde{S}$ for each $s$. We now consider several possibilities of how $\beta\left(T^{2}\right)$ intersects the $S$.

If $\beta\left(T^{2}\right)$ is disjoint from $S$ then either $\beta\left(T^{2}\right)$ lies entirely in $Q_{0}$ or else it lies entirely in $P_{0}$. If $\beta\left(T^{2}\right)$ lies entirely in $Q_{0}$ then $\alpha$ and $t^{s} \alpha$ have images in different copies of $Q_{0}$ lying above $Q_{0}$ and so the intersection number $\left(t^{s}+t^{-s}\right) \alpha \cdot \alpha_{0}$ vanishes for $s \neq 0$. 


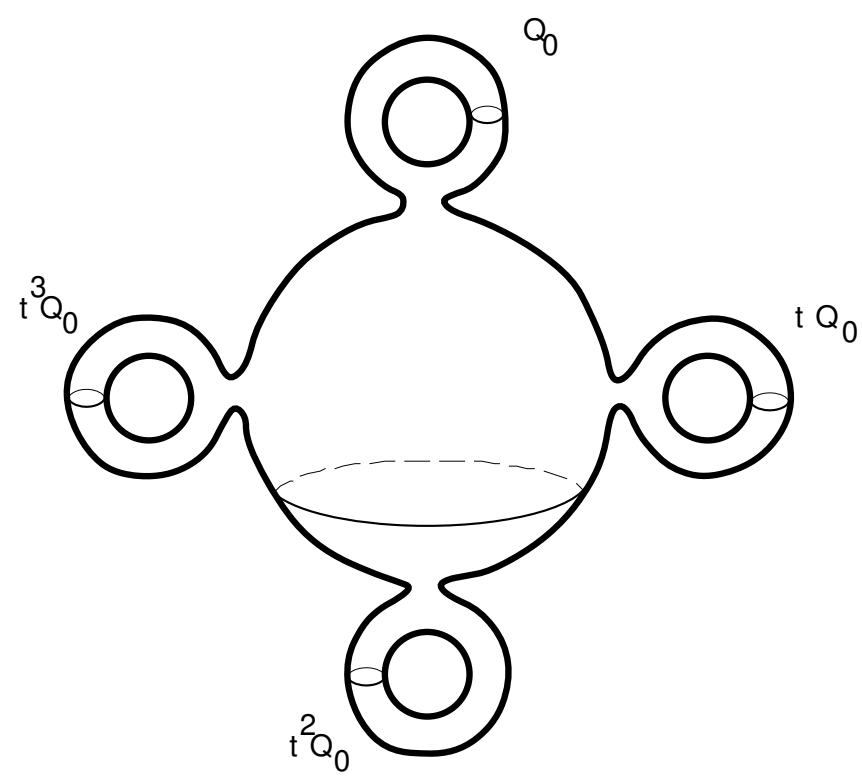

Figure 4.

If $\beta\left(T^{2}\right)$ lies entirely in $P_{0}$, then fill in the boundary of $P_{0}$ to obtain $P$ by attaching a 3-ball and extend $\tau$ over $P$. Also attach $n 3$-balls to $\tilde{P}_{0}$ to obtain the corresponding cover $\tilde{P}$.

If $\beta: T^{2} \longrightarrow P$ is homotopic into the boundary of $P$, then $\alpha$ is homotopic into the boundary of $\tilde{P}$ and using a collar of the boundary one sees easily that $\left(t^{s}+t^{-s}\right) \alpha \cdot \alpha_{0}$ vanishes. If $\pi_{1} P$ is finite, then so is $\pi_{1} \tilde{P}$, and hence the curve $\alpha_{0}$ is nullhomologous. This implies that $\left(t^{s}+t^{-s}\right) \alpha \cdot \alpha_{0}$ vanishes.

If $\pi_{1} P$ is infinite and $\beta: T^{2} \longrightarrow P$ is not injective on fundamental groups, then $\alpha: T^{2} \longrightarrow \tilde{P}$ is not injective on fundamental groups and since $\left.\pi_{(} \tilde{P}\right)$ is torsion free $\alpha$ can be compressed. This implies that the homology class carried by $\alpha$ in $H_{2}(\tilde{P} ; \mathbf{Z})$ is represented by a spherical class, which must vanish since $\pi_{2}(\tilde{P})=0$.

This leaves the case when $\beta: T^{2} \longrightarrow P$ is essential. This case is covered by Lemma 4.3 above.

Next we study the situation when $\beta: T^{2} \longrightarrow N$ intersects $S$. If every curve in $\beta^{-1}(S)$ is inessential in $T^{2}$, then by a homotopy of $\beta$ we may assume that $\beta\left(S^{1} \times *\right)$ misses $S$, and then by working with innermost circles we may surger $\alpha\left(T^{2}\right)$ to obtain a different representative of the homology class $\alpha$ which is given by a map of a union of a torus containing $\alpha_{0}$ and a union of 2-spheres; so

$$
\alpha \sim \alpha^{\prime}: T^{2} \cup_{i} S_{i}^{2} \longrightarrow \tilde{N}-\left(\cup_{s} t^{s} \tilde{S}\right)
$$


If $\alpha^{\prime}\left(T^{2}\right)$ is contained in the preimage of $Q_{0}$, then $\alpha_{0}$ has image in the preimage of $Q_{0}$ and since each component in this preimage has first homology trivial, $\left(t^{s}+t^{-s}\right) \alpha \cdot \alpha_{0}$ vanishes. The case when $\alpha^{\prime}\left(T^{2}\right)$ is contained in $\tilde{P}_{0}$ is identical to the argument given above.

The only remaining case to consider is if $\beta^{-1}(S)$ contains an essential curve in $T^{2}$. In this case we surger both $\alpha$ and the curve $\alpha_{0}$ simultaneously, obtaining a map $\alpha^{\prime}$ homologous to $\alpha$ from a union of 2 -spheres into the complement of the $t^{s} \tilde{S}$

$$
\alpha \sim \alpha^{\prime}: \cup_{i} S_{i}^{2} \longrightarrow \tilde{N}-\left(\cup_{s} \tilde{S}\right)
$$

and a union of curves in the complement of the $t^{s} \tilde{S}$ homologous to $\alpha_{0}$

$$
\alpha_{0} \sim \alpha_{0}^{\prime}: \cup_{j} S_{j}^{1} \longrightarrow \tilde{N}-\left(\cup_{s} \tilde{S}\right) .
$$

This time the expression $\left(t^{s}+t^{-s}\right) \alpha \cdot \alpha_{0}=\left(t^{s}+t^{-s}\right) \alpha^{\prime} \cdot \alpha_{0}^{\prime}$ vanishes because each intersection either takes place in some component of the preimage of $Q_{0}$ which has first homology trivial, or in the cover $\tilde{P}_{0}$, in which every 2 dimensional spherical homology class is zero. This completes the proof of the Theorem.

\section{References}

[Ba] D. Bar-Natan, On the Vassiliev knot invariants, Topology, 34 (1995), 423-472.

[B] J. Birman, New points of view in knot theory, Bulletin of the A.M.S., 28(2) (1993), 253-287.

[BL] J. Birman and X.-S. Lin, Knot polynomials and Vassiliev's invariants, Invent. Math., 111 (1993), 225-270.

[CJ] A. Casson and D. Jungreis, Convergence groups and Seifert-fibered 3-manifolds, Invent. Math., 111 (1993), 441-456.

[G] D. Gabai, Convergence groups are fuchsian groups, Annals of Math., 136(2) (1992), 447-510.

[He] J. Hempel, 3-manifolds, Annals of Math. Studies, 86, Princeton University Press, Princeton, New Jersey, 1976.

[JS] W. Jaco and P. Shalen, Seifert-fibered spaces in 3-manifolds, Memoirs of the A.M.S., Vol 21, 220, (1979).

[Jo] K. Johannson, Homotopy equivalences of 3-manifolds with boundaries, Springer LNM, 761, Springer Verlag.

[Ka] E. Kalfagianni, Finite type invariants for knots in 3-manifolds, Topology, 37(3) (1998), 673-707.

[KL] P. Kirk and C. Livingston, Type 1 invariants in 3-manifolds, Pacific J. Math., 183(2) (1998), 305-331.

[Li] X.-S. Lin, Finite type link invariants of 3-manifolds, Topology, 33 (1994), 45-71.

[O] T. Ohtsuki, Finite type invariants of integral homology 3-spheres, J. Knot Theory Ramifications, 5(1) (1996), 101-115. 
[S] T. Stanford, The functoriality of Vassiliev-type invariants of links, braids, and knotted graphs, J. Knot Theory Ramifications, 3 (1994), 247-262.

[V] V. Vassiliev, On invariants and homology of spaces of knots in arbitrary manifolds, MSRI Preprint \#1997-024.

Received July 15, 1998 and revised September 20, 1999.

INDIANA UNIVERSITY

Bloomington, IN 47405

E-mail address: pkirk@indiana.edu

INDIANA UNIVERSITY

Bloomington, IN 47405

E-mail address: livingst@indiana.edu 